ANNALES

POLONICI MATHEMATICI

$84.3(2004)$

\title{
Holomorphic series expansion of functions of Carleman type
}

\author{
by TAiB Belghiti (Kénitra)
}

\begin{abstract}
Let $f$ be a holomorphic function of Carleman type in a bounded convex domain $D$ of the plane. We show that $f$ can be expanded in a series $f=\sum_{n} f_{n}$, where $f_{n}$ is a holomorphic function in $D_{n}$ satisfying $\sup _{z \in D_{n}}\left|f_{n}(z)\right| \leq C \varrho^{n}$ for some constants $C>0$ and $0<\varrho<1$, and where $\left(D_{n}\right)_{n}$ is a suitably chosen sequence of decreasing neighborhoods of the closure of $D$. Conversely, if $f$ admits such an expansion then $f$ is of Carleman type. The decrease of the sequence $D_{n}$ characterizes the smoothness of $f$.
\end{abstract}

1. Introduction. Let $\left(M_{n}\right)_{n \geq 0}$ be an increasing sequence of positive real numbers. There exist two ways to define that a given $\mathcal{C}^{\infty}$ function, $f$, on an interval $[a, b] \subset \mathbb{R}$, belongs to the regular Carleman class $\mathcal{C}\left(M_{n}\right)$. First, there exist positive constants $C$ and $\varrho$, depending on $f$, such that $\left|f^{(n)}(x)\right| \leq C \varrho^{n} M_{n}$ for all $x \in[a, b]$ and $n \in \mathbb{N}$. (See [Ko], [Ma].) Second, $f$ admits an extension, $F$, not unique, to the whole complex plane such that $\bar{\partial} F$ decreases rapidly near $[a, b]$; here $\bar{\partial}=\frac{1}{2}(\partial / \partial x+i \partial / \partial y)$ is the Cauchy-Riemann operator. (See [Dy] and below.)

The purpose of this short note is to characterize the holomorphic functions on a bounded convex domain $D$ belonging to a given Carleman class as those functions that can be expanded in a series of functions holomorphic in an appropriate sequence of decreasing neighborhoods of the closure of $D$, satisfying some growth estimates. The decrease of these neighborhoods is directly linked with the given class.

The first result of this kind was obtained by J. C. Tougeron [To, 2.7-2.9] in the particular case where $D$ is a bounded sector and $M_{n}=(n !)^{k}$, with $k>1 / 2$, which corresponds to a Gevrey class. Our approach is different and may be extended to sets which are Whitney regular.

2000 Mathematics Subject Classification: 30H05, 30B10.

Key words and phrases: Carleman class, holomorphic expansion. 
2. The class $\mathcal{H}_{M}(D)$. Let $D$ be a bounded convex domain in the plane; $\mathcal{O}(D)$ and $\mathcal{O}(\bar{D})$ are spaces of holomorphic functions on $D$ and in a neighborhood of $\bar{D}$ respectively.

Let $M=\left(M_{n}\right)_{n}$ be an increasing sequence of positive real numbers. Let $\mathcal{H}_{M}(D)$ be the class of all functions $f \in \mathcal{O}(D)$ such that, for some positive constants $C$ and $\varrho$,

$$
\sup _{z \in D}\left|f^{(n)}(z)\right| \leq C \varrho^{n} M_{n}, \quad n \gg 0 .
$$

Note that every function $f$ belonging to $\mathcal{H}_{M}(D)$ can be extended to a $\mathcal{C}^{\infty}$ function on $\bar{D}$ : if $w \in \partial D$ and if $z_{p} \in D$ converges to $w$, the sequence $f^{(n)}\left(z_{p}\right)$ converges (because $f^{(n+1)}$ is bounded on $D$; apply the mean value theorem). We denote this extension also by $f$.

In order to get classes of holomorphic functions with structural properties and to have precise computations, we start, following [El], from a sequence $\left(M_{n}\right)_{n}$ such that $M_{n}:=M(n)$ with $M(t)=e^{m(t)}$ and $m(t)=t \log t+t \mu(t)$. Throughout the paper $\mu(t)$ will be a strictly increasing $\mathcal{C}^{\infty}$ function defined for $t \gg 0$ such that $\lim _{t \rightarrow+\infty} \mu(t)=+\infty$ (so $\left.\mu^{\prime}(t)>0\right)$. We also suppose that $\mu(t)$ belongs to a Hardy field (i.e a field of germs of functions at $+\infty$ in $\mathbb{R}$ which is closed under differentiation) and $\mu(t) \leq a t, t \gg 0, a>0$. This ensures that our class is an algebra closed under differentiation (the proof is easy and it is the same as in the real case, see $[\mathrm{El}])$ and strictly contains $\mathcal{O}(\bar{D})$.

Notice finally that the above class does not change if we replace $M(t)$ by $C \varrho^{t} M(t)$ where $C>0$ and $\varrho>0$. Consequently, $\mu$ is defined modulo an additive constant.

3. The functions $\Omega(s)$ and $\Gamma(u)$. Set

$$
\Omega(s):=\inf _{t \geq t_{0}} s^{-t} e^{t \mu(t)}, \quad s \gg 0,
$$

where $t_{0}>0$ is fixed. The infimum is attained when $t \mu^{\prime}(t)+\mu(t)=\log s$. The function $t \mu^{\prime}(t)+\mu(t)$ tends to infinity as $t \rightarrow+\infty$ and so it is strictly increasing $(\mu(t)$ belongs to a Hardy field); so we have a unique value of $t$ where the infimum is attained. Thus, if $\Omega(s)=e^{-\omega(s)}$, then we get the system

$$
s=e^{t \mu^{\prime}(t)+\mu(t)}, \quad \omega(s)=t^{2} \mu^{\prime}(t) .
$$

Since $\mu^{\prime}(t)>0$, we have $\omega(s)>0$ and $\lim _{s \rightarrow+\infty} \omega(s)=+\infty$. Thus, $\Omega(s)$ is strictly decreasing and $\lim _{s \rightarrow \infty} \Omega(s)=0$.

Set $\Gamma(u):=e^{-\gamma(u)}$, where $u$ and $\gamma(u)$ are defined by

$$
u=t^{2} \mu^{\prime}(t), \quad \gamma(u)=t \mu^{\prime}(t)+\mu(t) .
$$

As $\mu(t)$ is strictly increasing and $\lim _{t \rightarrow+\infty} \mu(t)=+\infty$, it follows that $\gamma(u)$ is strictly increasing and $\lim _{u \rightarrow+\infty} \gamma(u)=+\infty$. Hence, $\Gamma(u)$ is strictly de- 
creasing and $\lim _{u \rightarrow+\infty} \Gamma(u)=0$. The system (2) gives easily

$$
t=1 / \gamma^{\prime}(u), \quad \mu(t)=\gamma(u)-u \gamma^{\prime}(u),
$$

which shows that $\gamma^{\prime}$ is strictly decreasing, positive and $\lim _{u \rightarrow+\infty} \gamma^{\prime}(u)=0$. Notice that $\gamma(u)$, just as $\mu(t)$, is defined modulo an additive constant.

4. Main result. Define

$$
D_{n, R}^{\Gamma}:=\{z \in \mathbb{C} ; d(z, D)<R \Gamma(n)\} .
$$

Under the condition $\lim _{t \rightarrow+\infty}(\log t) / \mu(t) \neq 0$, we have the following:

Theorem 1. (a) Let $f \in \mathcal{H}_{M}(D)$. Then there exist $R>0, C>0$, $0<\varrho<1$, and a sequence $\left(f_{n}\right)_{n}$ with $f_{n} \in \mathcal{O}\left(D_{n, R}^{\Gamma}\right)$ such that:

(i) $\left\|f_{n}\right\|_{D_{n, R}^{\Gamma}} \leq C \varrho^{n}$ for all $n$;

(ii) $\sum_{n} f_{n}=f$ uniformly on $\bar{D}$.

(b) Conversely, let $R>0$ and let $f_{n} \in \mathcal{O}\left(D_{n, R}^{\Gamma}\right)$ be such that $\left\|f_{n}\right\|_{D_{n, R}^{\Gamma}}$ $\leq C \varrho^{n}$ for some constants $C>0$ and $0<\varrho<1$, and for all $n \geq n_{0}$ $\left(n_{0}\right.$ fixed). Then $f:=\sum_{n} f_{n}$ belongs to the class $\mathcal{H}_{M}(D)$.

5. Technical lemmas. With the above notations we have

LEMma 1. The function $\omega(s)$ is the inverse, under composition, of the function $e^{\gamma(u)}=1 / \Gamma(u)$, i.e. $\omega(s)=\gamma^{-1}(\log s), s \gg 0$.

Proof. It suffices to compare the systems (1) and (2).

Let us introduce the class $\mathcal{H}_{M_{\alpha}}(D), \alpha>0$, which corresponds to $\mu(\alpha t)$, i.e. we replace $\mu(t)$ by $\mu_{\alpha}(t)=\mu(\alpha t)$. So $M_{\alpha}(t)=e^{m_{\alpha}(t)}$, where $m_{\alpha}(t)=$ $t \log t+t \mu(\alpha t)$. Let $\Omega_{\alpha}(s)=e^{-\omega_{\alpha}(s)}$ and $\Gamma_{\alpha}(u)=e^{-\gamma_{\alpha}(u)}$ be the corresponding functions of the class $\mathcal{H}_{M_{\alpha}}(D)$. We have the following:

Lemma 2. For all $\alpha>0$,

(i) $\omega_{\alpha}(s)=(1 / \alpha) \omega(s)$,

(ii) $\gamma_{\alpha}(u)=\gamma(\alpha u)$.

Proof. By (2), we have $u=t^{2} \alpha \mu^{\prime}(\alpha t)$ and $\gamma_{\alpha}(u)=t \alpha \mu^{\prime}(\alpha t)+\mu(\alpha t)$; so if $\widetilde{t}:=\alpha t$, then $\alpha u=\widetilde{t} \mu^{\prime}(\widetilde{t})$ and $\gamma_{\alpha}(u)=\widetilde{t} \mu^{\prime}(\widetilde{t})+\mu(\widetilde{t})$. Thus we have (ii). Using Lemma 1 and (ii) we get

$$
\omega_{\alpha}(s)=\gamma_{\alpha}^{-1}(\log s)=\frac{1}{\alpha} \gamma^{-1}(\log s)=\frac{1}{\alpha} \omega(s) .
$$

Lemma 3. If $\lim _{t \rightarrow+\infty}(\log t) / \mu(t) \neq 0$, then $\mathcal{H}_{M_{\alpha}}(D)=\mathcal{H}_{M}(D)$.

Proof. By assumption, there exists $A>0$ such that $\mu(t) \leq A \log t$ for $t \gg 0$; then $t \mu^{\prime}(t) \leq B$ for some constant $B>0$. Hence $|\mu(\alpha t)-\mu(t)| \leq$ 
$|1-\alpha| t \mu^{\prime}(t) \leq B|1-\alpha|$. Thus $\mu(\alpha t)-\mu(t)$ is bounded. But $\mu$ is defined modulo an additive constant, so we can choose $\mu_{\alpha}=\mu$.

6. Proof of Theorem 1. Let $f \in \mathcal{H}_{M}(D)$. By Lemma $3, f \in \mathcal{H}_{M_{\alpha}}(D)$ for every $\alpha>0$. With the help of Dynkin's scheme (see [Dy, pp. 41-43] adjusted to our situation, $f$ can be extended to a $\mathcal{C}^{\infty}$ function on the whole plane, say $F$, with compact support and such that the following estimate holds:

$$
\left|\frac{\partial F}{\partial \bar{\zeta}}(\zeta)\right| \leq C_{2} e^{-\omega_{\alpha}\left(1 / C_{1} d(\zeta, D)\right)}
$$

for every $\zeta \in \mathbb{C}-\bar{D}$. In the above $C_{1}$ and $C_{2}$ are positive constants depending on $\alpha$ and $f ; d(\zeta, D)$ is the Euclidean distance from $\zeta$ to $D$; and $\partial / \partial \bar{\zeta}$ is the Cauchy-Riemann operator. Fix such an $F$ with $\operatorname{supp} F \subset$ $\{\zeta \in \mathbb{C} ; d(\zeta, D)<r\}$, where $r$ is a fixed positive number; and let $R=$ $r / \Gamma\left(n_{0}\right)$, where $n_{0}$ is an integer to be chosen later. Now, set

$$
D_{n}:=D_{n, R}^{\Gamma}=\left\{\zeta \in \mathbb{C} ; d(\zeta, D)<r \frac{\Gamma(n)}{\Gamma\left(n_{0}\right)}\right\}, \quad n \geq n_{0} .
$$

Notice that $\operatorname{supp} F \subset D_{n_{0}} ; D_{n}$ is an open convex neighborhood of $\bar{D}$; $D_{n+1} \subset D_{n}$ for all $n$; and $\bigcap_{n \geq n_{0}} D_{n}=\bar{D}$.

Let $f_{n}$ be the $\mathbb{C}$-valued function defined for every $z \in D_{n}$ by

$$
f_{n}(z)=\frac{-1}{\pi} \int_{D_{n-1} \backslash D_{n}} \frac{\frac{\partial F}{\partial \bar{\zeta}}(\zeta)}{\zeta-z} d \xi d \eta, \quad \zeta=\xi+i \eta, n \geq n_{0} .
$$

Clearly $f_{n} \in \mathcal{O}\left(D_{n}\right)$ and since $F(=f)$ is holomorphic on $D$, by the CauchyGreen formula we have

$$
\begin{aligned}
f(z) & =\frac{-1}{\pi} \int_{C} \frac{\frac{\partial F}{\partial \bar{\zeta}}(\zeta)}{\zeta-z} d \xi d \eta=\frac{-1}{\pi} \int_{D_{n_{0}} \backslash \bar{D}} \frac{\frac{\partial F}{\partial \bar{\zeta}}(\zeta)}{\zeta-z} d \xi d \eta \\
& =\sum_{n \geq n_{0}+1} \frac{-1}{\pi} \int_{D_{n-1} \backslash D_{n}} \frac{\frac{\partial F}{\partial \bar{\zeta}}(\zeta)}{\zeta-z} d \xi d \eta=\sum_{n \geq n_{0}+1} f_{n}(z), \quad z \in D .
\end{aligned}
$$

Next, by the estimate (4) on $\partial F / \partial \bar{\xi}$, by Lemmas $1-3$, choosing $n_{0}$ equal to the integer part of $\Gamma^{-1}\left(r C_{1}\right)$ where $\Gamma^{-1}$ is the inverse of the function $\Gamma$, we have

$$
\begin{aligned}
\left|\frac{\partial F}{\partial \bar{\zeta}}(\zeta)\right| & \leq C_{2} \exp \left(-\frac{1}{\alpha} \omega\left(\frac{1}{C_{1} d(\zeta, D)}\right)\right) \leq C_{2} \exp \left(-\frac{1}{\alpha} \omega\left(\frac{\Gamma\left(n_{0}\right)}{r C_{1} \Gamma(n-1)}\right)\right) \\
& \leq C_{2} \exp \left(-\frac{1}{\alpha} \omega\left(\frac{1}{\Gamma(n-1)}\right)\right)=C_{2} e^{-(n-1) / \alpha}
\end{aligned}
$$


for every $\zeta \in D_{n-1} \backslash D_{n}$. Otherwise, a proof similar to that of the AhlforsBeurling inequality (see [Ra, pp. 141-142]) gives the estimates

$$
\int_{D_{n-1} \backslash D_{n}} \frac{1}{|\zeta-z|} d \xi d \eta \leq \sqrt{\pi \operatorname{area}\left(D_{n_{0}}\right)} .
$$

Now, taking $C=C_{2} e^{1 / \alpha} \sqrt{\pi \operatorname{area}\left(D_{n_{0}}\right)}$ and $\varrho>e^{-1 / \alpha}$ we get $\left\|f_{n}\right\|_{D_{n}} \leq C \varrho^{n}$; and the proof of Theorem 1(a) is finished.

To prove the converse, let $z \in D$. Since the closed disc $\bar{D}(z,(R / 2) \Gamma(n))$ is contained in $D_{n}:=D_{n, R}^{\Gamma}(R>0$ is given), we use Cauchy's inequalities to get

$$
\frac{\left|f_{n}^{(p)}(z)\right|}{p !} \leq C \varrho^{n}\left(\frac{2}{R \Gamma(n)}\right)^{p}, \quad p=0,1, \ldots, n \geq 0 .
$$

Choose $\varrho^{\prime}$ such that $\varrho<\varrho^{\prime}<1$; then

$$
\sup _{z \in D} \frac{\left|f_{n}^{(p)}(z)\right|}{p !} \leq C\left(2 R^{-1}\right)^{p}\left(\frac{\varrho}{\varrho^{\prime}}\right)^{n} \varrho^{\prime n} \sup _{u>0} \frac{\varrho^{\prime u}}{(\Gamma(u))^{p}}, \quad p=0,1, \ldots, n \geq 0 .
$$

By summing the preceding inequalities over $n$ we get

$$
\begin{aligned}
\sup _{z \in D} \frac{\left|f^{(p)}(z)\right|}{p !} & \leq C\left(2 R^{-1}\right)^{p}\left(\frac{\varrho}{\varrho^{\prime}}\right)^{n_{0}} \frac{\varrho^{\prime}}{\varrho^{\prime}-\varrho} \sup _{u>0} \frac{\varrho^{\prime u}}{(\Gamma(u))^{p}} \\
& =C\left(2 R^{-1}\right)^{p}\left(\frac{\varrho}{\varrho^{\prime}}\right)^{n_{0}} \frac{\varrho^{\prime}}{\varrho^{\prime}-\varrho} \sup _{u>0} e^{\left\{u \log \varrho^{\prime}+p \gamma(u)\right\}}, \quad p=0,1, \ldots
\end{aligned}
$$

Furthermore the supremum is reached when $\gamma^{\prime}(u)=-\left(\log \varrho^{\prime}\right) / p$ and it is equal to $\exp \left\{p\left(\gamma(u)-u \gamma^{\prime}(u)\right)\right\}$. So, $f$ belongs to the class such that $\mu(p)=\gamma(u)-u \gamma^{\prime}(u)$. Thus, by $(3), f \in \mathcal{H}_{M_{\alpha}}(D)$ with $\alpha=-1 / \log \varrho^{\prime}$. Consequently, by Lemma $3, f \in \mathcal{H}_{M}(D)$ and the proof of Theorem 1 is complete.

TheOREM 1 FOR $\mathcal{C}(M(n))$. Let $f \in \mathcal{C}^{\infty}([a, b])$. Then $f \in \mathcal{C}(M(n))$ if and only if there exist constants $C>0,0<\varrho<1, R>0$ and a sequence of functions $f_{n}$, holomorphic in $E_{n}:=\{z \in \mathbb{C} ; d(z,[a, b])<R \Gamma(n)\}$ such that $\left\|f_{n}\right\|_{E_{n}} \leq C \varrho^{n}$ and $\sum_{n} f_{n}=f$ uniformly on $[a, b]$.

Note that our result is valid for the class $\mathcal{C}(M(n))$ whether or not the class is quasianalytic.

EXAmPLES. 1. $\mu(t)=\frac{1}{k} \log t, k>0$, which corresponds to the Gevrey class of order $k$. From (3) we obtain $u=\frac{1}{k} t$ and

$$
\gamma(u)=\frac{1}{k} \log t+\frac{1}{k}=\frac{1}{k}(\log u+\log k)+\frac{1}{k} ;
$$

so we can choose $\gamma(u)=\frac{1}{k} \log u$. 
2. $\mu(t)=\beta \log \log t(\beta>0)$. We obtain $u=\beta t / \log t$, so $\log u \sim \log t$, and

$$
\gamma(u)=\beta \log \log t+\frac{\beta}{\log t} ;
$$

so we can choose $\gamma(u)=\beta \log \log u$.

3. $\mu(t)=a t, a>0$ (extreme case); $\gamma(u)=2 \sqrt{a u}$.

Remarks. The condition $\mu(t) \leq a t$ implies that every function $\Gamma(u)$ is lower bounded by $e^{-2 \sqrt{a u}}$ at infinity, for some $a \gg 0$. Consequently, $\Gamma(u)$ is always subexponentially decreasing.

2. We can say more on the link between the function $M(t)=t^{t} e^{t \mu(t)}$, which ensures the growth of the derivatives, and the function $\Gamma(u)=e^{-\gamma(u)}$, which ensures the decrease of the neighborhoods $D_{n}$ : if $\lim _{t \rightarrow+\infty}(\log t) / \mu(t)$ $\neq 0$ we can choose $\gamma=\mu$ as in Examples 1 and 2 .

\section{References}

[Dy] E. M. Dynkin, The pseudo-analytic extension of smooth functions. The uniform scale, in: Amer. Math. Soc. Transl. (2) 115, 1980, 33-58.

[El] A. Elkhadiri, The theorem of the complement for a quasi-subanalytic set, Studia Math. 161 (2004), 225-247.

[Ko] H. Komatsu, The implicit function theorem for ultradifferentiable mapping, Proc. Japan Acad. Ser. A 55 (1979), 69-72.

[Ma] S. Mandelbrojt, Analytic functions and classes of infinitely differentiable functions, Rice Inst. Pamphlet 29 (1942) (reprinted in: Selecta, Gauthier-Villars, 1981).

[Ra] T. Ransford, Potential Theory in the Complex Plane, London Math. Soc. Student Texts 28, Cambridge Univ. Press, 1995.

[To] J. C. Tougeron, Sur les ensembles semi analytiques avec conditions Gevrey au bord, Ann. Sci. École Norm. Sup. 27 (1994), 173-208.

Department of Mathematics

Faculty of Sciences

B.P. 133, Kénitra, 14000, Morocco

E-mail: belghititaib@yahoo.fr

Reçu par la Rédaction le 3.7.2004

Révisé le 10.11.2004 